\title{
LA DECORAZIONE SCULTOREA NEI CHIOSTRI DELL'ITALIA MERIDIONALE COME VEICOLO DI RIFLESSIONE. IL CASO DEL CHIOSTRO DI SANTA SOFIA A BENEVENTO
}

\section{MARIA CRISTINA ROSSI}

UDC: $726.54(450.25)$

73.046 .3

Preliminary communication

Manuscript received: 24. 12. 2016.

Revised manuscript accepted: 23. 01. 2017.

DOI: 10.1484/J.HAM.5.113722

\author{
M. C. Rossi \\ Università "G. D’Annunzio" \\ di Chieti, 31 \\ 66100 Chieti \\ Italia
}

The cloister of Santa Sofia in Benevento, of Lombard foundation, saves a group of capitals carved with various subjects. The main theme of the decoration is directed to the contemplation for the monastic community: scenes from the Old and New Testament stories, scenes from the monastic environment, scenes from the profane world. The decoration of the cloister is therefore a didactic path for the monks. The narrative that is seen in the capitals of the cloister of Santa Sofia in Benevento is one of the most important in South Italy, especially for the presence of the cycle of the months that finds the strongest iconographic comparisons with North Italy. But the realization of the sculptures is linked to a workshop of local artists in the late twelfth century, influenced by apulian and from the Abruzzo figurative culture.

Keywords: cloister, iconography, capitals, monastery, benedictine

Nell'architettura monastica il chiostro era lo spazio a corte che si trovava chiuso tra la chiesa e l'organismo residenziale, costituito di gallerie aperte che correvano lungo il perimetro dell'impianto, solitamente a pianta quadrangola$\mathrm{re}^{1}$. Era il luogo dedicato alla preghiera e alla meditazione dei singoli all'interno della comunità religiosa ${ }^{2}$. Per tale ragione, l'arredo scultoreo, condensato solitamente nei capitelli delle colonnine che ritmavano il cortile, presentava specifici programmi decorativi, per lo più con funzioni escatologiche ricavate da scene del Vecchio e del Nuovo Testamento, dal mondo fantasioso dei bestiari, talvolta pure da iconografie proprie del contesto monastico, con episodi esaltanti la Regola, ma anche da brani mitologici e profani con chiari intenti metaforici.

L'ideatore delle forme architettoniche benedettine si sarebbe ispirato alle norme che regolavano la vita del monastero: anche il chiostro partecipava, come spazio, alle consuetudini quotidiane del monaco3 ${ }^{3}$, sebbene la Regola di san Benedetto non abbia comunque tramandato uno specifico programma edilizio funzionale al luogo dove si raccoglieva la vita comunitaria ${ }^{4}$. Ad ogni modo, è certo che la funzione che rivestiva il repertorio scultoreo del chiostro aderiva a propositi didascalici, laddove alle immagini veniva affidato il compito di illustrare, attraverso figure, $\mathrm{i}$ testi sacri e talvolta anche la Regola. Vivere iuxta regulam e dunque "vivere secondo la regola" significava vivere secondo la spiritualità monastica e, nel cenobio, erano note più regole, che venivano lette e commentate come testi di parenesi monastica, dai quali trarre precetti spirituali. Tutte le fondazioni monastiche, sin dalle loro origini, si diedero dei codici di comportamento, generalmente scritti ${ }^{5}$. Nella Regola si raccolsero anche le cosiddette "consuetudini" o norme di costumi, che rispondevano alle esigenze pratiche e che organizzavano dettagliatamente la vita quotidiana. Originariamente considerate come norme complementari, nei periodi in cui la ritualizzazione della vita monastica toccò livelli di esasperazione, le consuetudini acquisirono grande importanza fino ad essere la modalità concreta con cui la Regola era applicata nel cenobio o all'interno della rete di monasteri appartenenti al medesimo ordine, come nel caso di Cluny.

La Regola benedettina fu redatta intorno alla metà del VI secolo ed è composta da un prologo iniziale e da settantatré capitoli $^{6}$. Vi si distinguono tre grandi blocchi, che sembrerebbero provenire da altrettanti periodi della redazione e corrispondenti ai momenti consueti dell'esperienza cenobitica e dell'itinerario spirituale. Il primo comprende il prologo e i primi sette capitoli, nei quali il legislatore tratta i temi dell'ascesa spirituale, definisce le ragioni del primato cenobitico e descrive il monastero come una scuola che educa al servizio di Dio. Il secondo, che va dal capitolo otto al sessantaseiesimo ed esamina i diversi istituti monastici: l'ufficio divino e la preghiera, il codice penitenziale, le varie attività quotidiane del monaco, l'accesso al monastero e le forme

\footnotetext{
${ }^{1}$ P. F. PISTILLI, s.v., Chiostro, in Enciclopedia dell'arte medievale, IV, Roma, 1993, p. 694.

${ }^{2}$ G. ARCHETTI, Spazi e strutture claustrali nei commenti carolingi alla regola benedettina, in Hortus artium medievalium, 20 (2014), p. 448-462: 450, 451. 3 G. ARCHETTI, Boscose solitudini. Simbolo, immagini e figure dal mondo monastico, in A. C. Quintavalle (a cura di), Medioevo natura e figura, Atti del Convegno internazionale di studi (Parma, 20-25 settembre 2011), Milano, 2015, p. 297-310: 299; IDEM, "Dilexi decorem domus tuae". Committenza aristocratica e popolare in ambito claustrale (secoli VIII-XII), in A. C. Quintavalle (a cura di), Il Medioevo: $i$ committenti, Atti del Convegno internazionale di studi (Parma, 21-26 settembre 2010), Milano, 2011 (I convegni di Parma, 13), p. 237-251.

${ }^{4}$ G. GIURISATO, Il cenobio di Benedetto: un'icona della chiesa, Bresseo di Teolo, 2011, p. 35-36.

5 GIURISATO, Il cenobio di Benedetto, p. 11, 12.

${ }^{6}$ GIURISATO, Il cenobio di Benedetto, p. 17, 21; S. PRICOCO, "Benedictus de Nursia". La Regola di San Benedetto e le regole dei Padri, Milano, 2006.
} 
di reclutamento. L'ultima parte, dal capitolo sessantasette al settantaduesimo, propone i fondamenti spirituali della prassi cenobitica. L'ultimo capitolo, il settantatré, indica le finalità della silloge normativa quale "piccola regola" o primo strumento adatto a tutti per avviarsi alla vita monastica. Si tratta dunque di un complesso di regole redatte per precisare le norme di vita della comunità monastica? ${ }^{7}$.

Con l'espressione ora et labora si sintetizza generalmente il significato della regola di san Benedetto rintracciabile nel capitolo quarantotto, in cui con l'esortazione labora s'intendono i compiti manuali che ripartivano in tre tempi la giornata del monaco. "L'ozio è nemico dell'anima, e perciò $i$ fratelli in determinate ore devono essere occupati in lavori manuali, in altre nella lettura divina"8. La meditazione nel chiostro, facilitata dalla "resa in figura" dei sacri testi e di altri desunti da varie fonti con il medesimo intento educativo, apparteneva a quest'ultima fase. Il lavoro, inteso come fatica fisica, già dalla tarda età longobarda, rientrava pienamente nella ritualità quotidiana 9 . Alla luce di queste considerazioni preliminari, vorrei presentare un caso significativo di "testo" reso per immagini, allestito nei capitelli del chiostro di Santa Sofia a Benevento.

Quando nel 774 Carlo Magno conquistò Pavia, la capitale del Regno longobardo, ponendo cosi fine alla dominazione dei Longobardi in Italia, non riusci a impossessarsi anche del Ducato di Benevento, comprendente gran parte della penisola italiana meridionale, ad eccezione di alcune aree della costa campana, della Puglia e della Calabria ${ }^{10}$. La politica del duca beneventano Arechi II, principe dell'antico ducato, mirava a una posizione fortemente autonoma all'interno del governo franco, una politica caratterizzata, peraltro, anche da una tendenza all'accoglienza di un insieme di culture diverse. La fucina di questa realtà era chiaramente la capitale: ipsa harum provinciarum caput ditissima Beneventus, come la definiva Paolo Diacono con toni encomiastici" ${ }^{11}$

La chiesa di Santa Sofia fu costruita nel secolo VIII per volere del duca Arechi II, sebbene la prima iniziativa si deve a Gisulfo II, che le conferì la dedicazione alla Santa Sapienza ${ }^{12}$. L'edificio fu distrutto da un fortissimo terremoto nel 968 e il nuovo chiostro fu realizzato con una forma quadrangolare, composto da quindici quadrifore e una trifora che nell'angolo sud, in corrispondenza della chiesa attigua, ripiegava con un

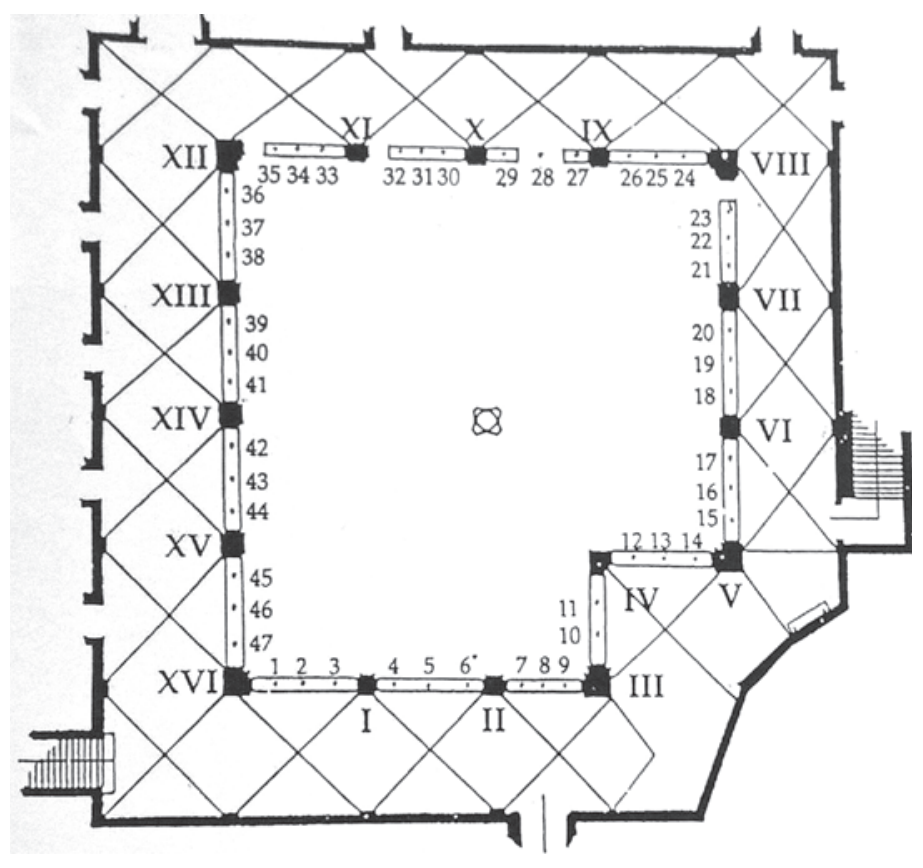

Fig. 1. Benevento, Santa Sofia, pianta del chiostro (Costagliola 2002)

angolo sporgente (Fig. 1). Alcuni frammenti della costruzione originaria furono reimpiegati nella nuova decorazione scultorea, mentre altri si trovano conservati nell'attiguo Museo del Sannio ${ }^{13}$. Sotto il duca Arechi fu fondato anche l'annesso monastero benedettino femminile, affidato inizialmente alla sorella dello stesso sovrano ${ }^{14}$. La Guida della città di Benevento stilata nel 1900 descrive il chiostro di Santa Sofia in tono molto partecipato: «Il chiostro del convento della chiesa di Santa Sofia contiguo alla detta chiesa, porge indicibile diletto con le sue arcate quadrifore, dagli archi moreschi che poggiano su colonnine, dai varii scolpiti capitelli, e più ancora perché può dirsi quasi unico nel suo genere non pure per la sua antichità; ma si veramente per avere l'arco a ferro di cavallo, a somiglianza dei tempii di Costantinopoli» ${ }^{15}$.

Di quella preziosità arricchita oltremodo da sfumature nostalgiche si teneva conto anche alla fine del XIX secolo, quando il chiostro fu restaurato a più riprese a partire dal $1878^{16}$. Qualche anno dopo, nel 1890 l'ispettore per i Monumenti di Benevento fece una urgente richiesta di restauro

7 G. Picasso (a cura di), San Benedetto. La regola, Cinisello Balsamo, 2009, p. 29-44.

8 Regula Benedicti 48, 1 in G. Picasso (a cura di), San Benedetto, p. 147; G. ARCHETTI, "Nihil operi Dei praeponatur". Il tempo dei monaci nel Medioevo, in “Tempus mundi umbra aevi". Tempo e cultura del tempo tra Medioevo e età moderna, Atti dell'incontro nazionale di studio (Brescia, 29-30 marzo 2007), a cura di G. Archetti e A. Baronio, Brescia, 2008 (Storia, cultura e società, 1), p. 51-80.

9 ARCHETTI, Spazi e strutture claustrali, p. 455; IDEM, Scuola, lavoro e impegno pastorale: l'abbazia di Leno nel Medioevo (secoli IX-XIV), in L'abbazia di San Benedetto di Leno. Mille anni nel cuore della pianura Padana, Atti della giornata di studio (Leno, Villa Seccamani, 26 maggio 2001 ), a cura di A. Baronio, Brescia, 2002 (Brixia sacra, VII, 1-2), p. 93-138.

10 L. A. BERTO, Piccola storia dei longobardi di Benevento, Napoli, 2013, p. 1.

"G. VERGINEO, Storia di Benevento e dintorni. Dalle origini mitiche agli Statuti del 123o, I, Benevento 1985, p. 112; M. Rotili (a cura di), Arechi II e il ducato di Benevento, Convegno internazionale di studi (Benevento, 15-17 maggio 2014), Padova, 2017 (Biennale di studi longobardi, 1), in stampa.

${ }^{12}$ M. ROTILI, Benevento, Chiesa di S. Sofia, in Corpus della scultura altomedievale, V. La diocesi di Benevento, Spoleto, 1966, p. 29, 31, 33; IDEM, I monumenti della Longobardia meridionale, in La civiltà dei Longobardi in Europa, Atti del Convegno Internazionale (Roma, Accademia Nazionale dei Lincei-Cividale del Friuli, 24-28 maggio 1971), Roma, 1971, p. 212-213; R. NALDI, Ritorno al chiostro di Santa Sofia a Benevento, in Bollettino d'Arte, VI, 76 (199o), p. 2566; VERGINEO, Storia di Benevento, p. 106; M. COSTAGLIOLA, Nuovi dati sulla chiesa longobarda di S. Sofia a Benevento, in III Congresso Nazionale di Archeologia Medievale (Castello di Salerno, Complesso di Santa Sofia, Salerno, 2-5 ottobre 2003), Firenze, 2003, p. 6oo-6o8: 6oo; V. PACE, Arte medievale in Italia meridionale, I. Campania, Napoli, 2007, p. 3; G. ARCHETTI, “Sequens vestigia regum”. Note conclusive, in M. Rotili (a cura di), Arechi II e il ducato di Benevento, in stampa.

13 COSTAGLIOLA, Nuovi dati sulla chiesa longobarda, p. 600.

14 VERGINEO, Storia di Benevento, p. 106.

15 Guida di Benevento, Benevento, 1990, p. 16.

${ }^{16}$ Archivio Centrale di Stato di Roma, AABBAA, I versamento, II serie, b. 434, fasc. 107. 


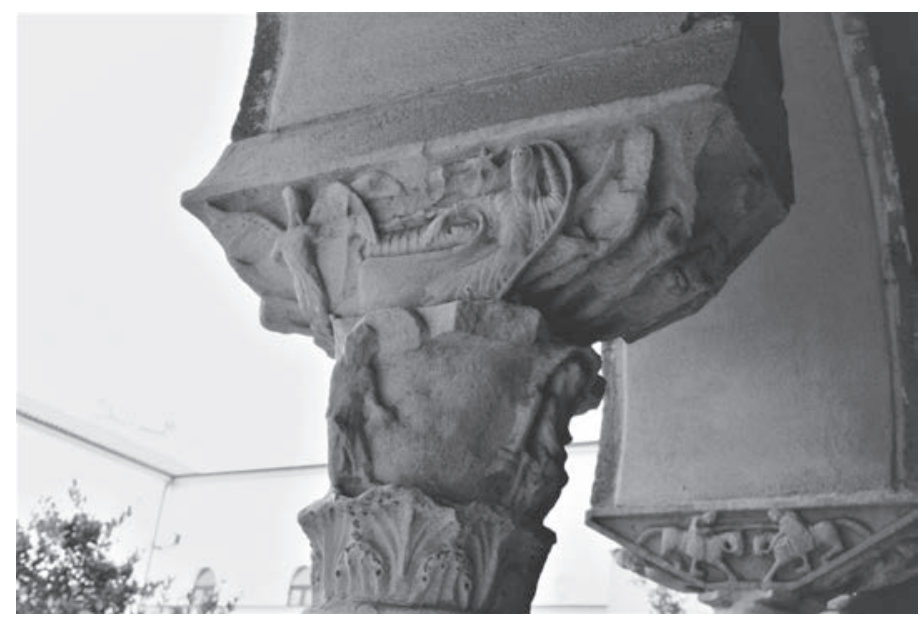

Fig. 2. Benevento, Santa Sofia, chiostro, capitello con La nascita di Gesù (Foto Margherita Tabanelli)

del chiostro. Quel luogo infatti era stato ceduto dal Comune alla comunità per ricavarne una scuola elementare, poi affidata ai padri Ignorantelli ${ }^{17}$. Proprio ai padri il ministero ai Monumenti della Provincia di Benevento chiedeva di provvedere al riparo dei danni degli archetti e dei capitelli del chiostro medievale, che erano stati sporcati con vernice bianca e con macchie di latte di calce dagli alunni che vi trascorrevano la ricreazione ${ }^{18}$. Ma già due anni prima i padri, in occasione della festa del fondatore del loro ordine, avevano provveduto alla sistemazione, tanto che nel 1890 l'ispettore per i monumenti di Benevento ne faceva menzione nella sua relazione: «per maggiore chiarimento aggiungerei che gli archetti antichi del chiostro non furono alterati». Altri interventi si registrarono nel 1896 sino alla metà del secolo successivo, quando nel 1956 il chiostro fu restaurato da Gino Chierici, provvedendo all'eliminazione di tutte le sovrapposizioni sul colonnato ${ }^{19}$.

Il chiostro di Santa Sofia, come si diceva di fondazione longobarda ${ }^{20}$, oggi sede del Museo del Sannio, si sviluppa su una pianta quadrata con il lato interno di circa 23 metri, quello esterno di circa $33^{21}$. Ė sostenuto da sedici pilastri, tra i quali si aprono quindici quadrifore e una trifora nell'angolo rientrante, formate da archetti a sesto acuto. Gli immediati confronti si rivolgono ai chiostri pugliesi coevi del monastero di San Benedetto a Bari, di San Benedetto a Conversano, in provincia di Bari e di San Benedetto a Brindisi, segnati dall'utilizzo della quadrifora e della trifora ${ }^{22}$. Nonostante le facili comparazioni ravvisabili con il disegno della struttura delle aperture tra il chiostro di Benevento e quello di Conversano, il confronto con la scultura dei capitelli con pulvino a stampella lascia spazio soltanto a un discorso di generale

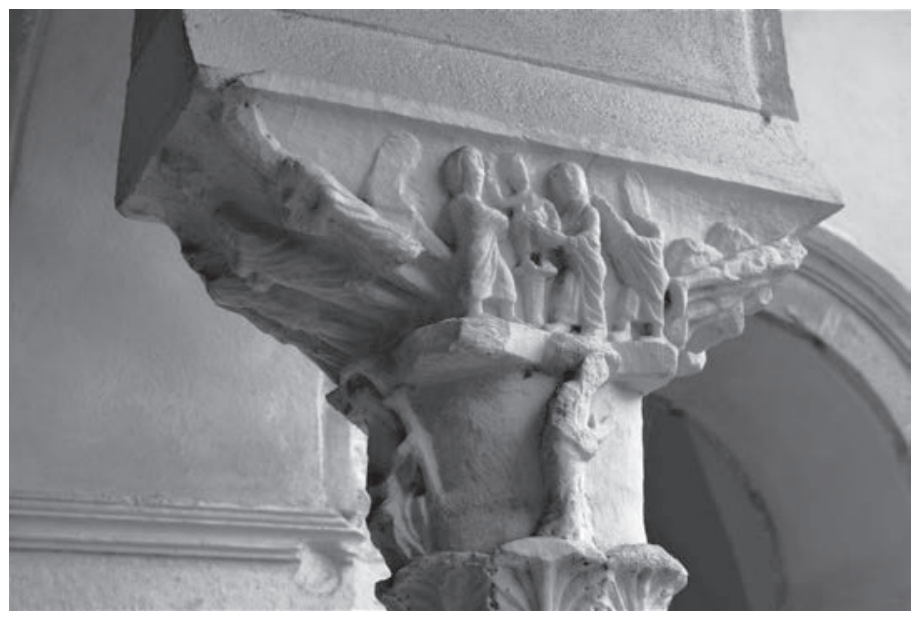

Fig. 3. Benevento, Santa Sofia, chiostro, capitello con La presentazione al tempio (Foto Margherita Tabanelli)

affiliazione del tratto decorativo riconducibile alla scultura di XII secolo dell'Italia meridionale.

Gli archetti poggiano su 47 colonnine, sormontate ciascuna da un capitello e un pulvino a stampella, variamente scolpiti. Il chiostro fu interamente ricostruito dopo i disastrosi terremoti del 1688 e del 1702, pertanto risulta ancora da indagare l'originaria disposizione dei capitelli, manomessa nel XVIII secolo durante i restauri promossi dal cardinale Vincenzo Maria Orsini, abate commendatario del complesso monastico di Santa Sofia ${ }^{23}$. In uno dei primi che si incontrano, troviamo scolpita una scena verosimilmente identificabile con San Benedetto assiso in cattedra che, rivolto a chi entrava nel chiostro, mostrava il libro della Regola, secondo un'iconografia tipica di quel genere. Sul pulvino ruotano scene della vita di Cristo: La nascita di Gesù, L'arrivo dei magi con $i$ doni e La presentazione di Gesù al tempio (Figg. $2,3)$. L'insieme viene generalmente attribuito al cosiddetto Maestro dei mesi, a capo della maestranza responsabile della scultura sofiana; su un capitello di spoglio furono scolpiti due mostri dalle code e dalle teste avvinghiate, attraverso una tecnica affine a un capitello dell'ambone quadrato della cattedrale di Salerno ${ }^{24}$.

Molto interessante si rivela la presenza del ciclo dei mesi. La rappresentazione dello svolgimento dell'anno attraverso figure, personificazioni o scene corrispondenti a ognuno dei dodici mesi costituisce un tema iconografico che affonda le sue origini nell'Antichità ${ }^{25}$. I mesi formano un ampio repertorio tematico, che, oltre a includere motivi religiosi, agricoli e folcloristici, si caratterizza per l'uso di un linguaggio figurativo che poteva variare dall'allegoria alla narrazione, mettendo comunque in risalto l'esposizione

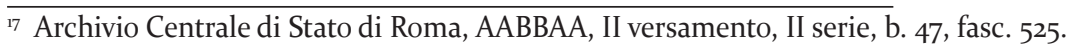

${ }^{18}$ Archivio Centrale di Stato di Roma, AABBAA, II versamento, II serie, b. 47, fasc. 525.

${ }^{19}$ COSTAGLIOLA, Nuove acquisizioni sui restauri novecenteschi, p. 32.

${ }^{20}$ E. SCHAFFRAN, Die Kunst der Langobarden in Italien, Jena, 1941, p. 26-31.

${ }^{21}$ E. GALASSO, Il chiostro di Santa Sofia a Benevento. Il simbolico, il mostruoso, l’ambiguo, Benevento, 1993, p. 15.

${ }^{22}$ P. BELLI D’ELIA, La Puglia in Italia Romanica, VIII, Milano, 1986, p. 200-206; M. LANERA, Appunti per la storia del monastero di San Benedetto di Conversano in Studi di Storia Pugliese in onore di Giuseppe Chiarelli, I, Galatina, 1972, p. 345-422.

${ }_{23}$ COSTAGLIOLA, Nuovi dati sulla chiesa longobarda, p. 6o1; I. BOVE, I restauri orsiniani al chiostro di S. Sofia a Benevento, in Rivista dell'Istituto Nazionale d'Archeologia e Storia dell'Arte, 3, 28 (2005), p. 193-212; la difficoltà di leggere la disposizione delle immagini distribuite lungo le gallerie del chiostro, a causa dei continui rimaneggiamenti, è stata messa in luce anche da V. DE DUONNI, Le sirene nei chiostri (e non più) della Campania, in Memoria Europae, II, 2 (2016), p. 52-77.

${ }^{24}$ L. COCHETTI, La decorazione plastica del chiostro di Santa Sofia, in Commentari, 8 (1957), p. 17-26: 20-21.

${ }^{25}$ M. A. CASTIÑEIRAS GONZÁLEZ, s.v., Mesi, in Enciclopedia dell'arte medievale, VIII, Roma, 1997, pp. 325-335.
} 


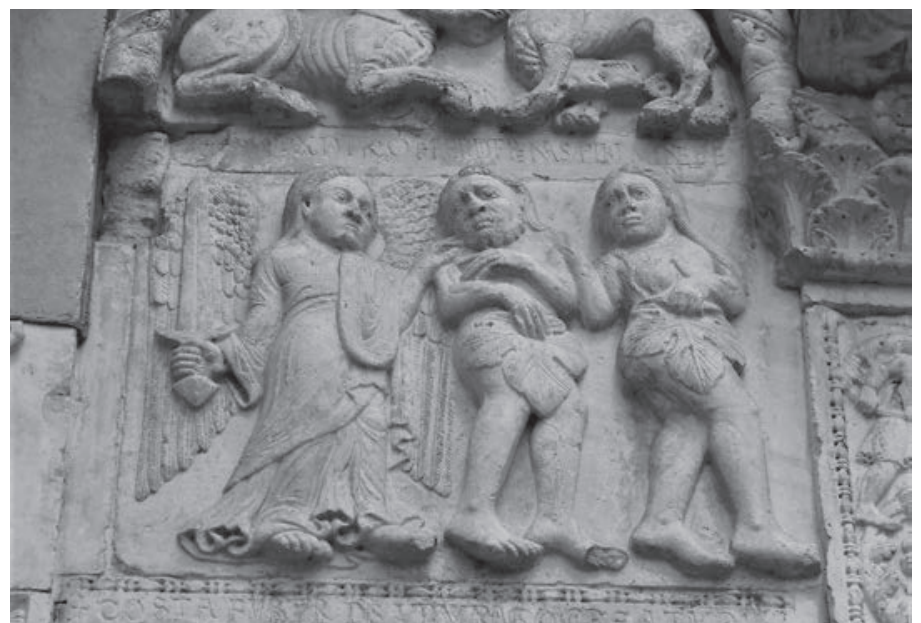

Fig. 4. Verona, San Zeno, portale maggiore, particolare della Cacciata di Adamo ed Eva (Foto Autore)

dell'anno agricolo con il corso dei lavori e degli intervalli di riposo.

Il ciclo deve essere interpretato, nel contesto monastico che si esamina, come indicatore del trascorrere del tempo della vita del cristiano, una sorta di cronometro, di orologio domestico; il lavoro dell'anno era consacrato a Dio e il tempo del lavoro umano era, per l'appunto, il tempo di $\mathrm{Dio}^{26}$. Non è un caso, infatti, che accanto al calendario compaia di solito un gruppo di temi ricorrenti e, in tal senso, ciclici per l'appunto, tra i quali s'incontrano spesso: la Creazione, la Storia di Adamo ed Eva e dei loro figli, la Natività, l'Epifania, il Battesimo di Cristo, il Giudizio universale, accanto a temi di carattere enciclopedico e di vari trattazione, come i segni dello zodiaco, i pianeti e le stagioni e soggetti della cultura profana, come i racconti cavallereschi inseriti in narrazioni storiche. Uno degli esempi di tal genere si ritrova nel celebre portale della chiesa di San Zeno a Verona, allestito secondo un programma iconografico arricchito dalle Storie del Vecchio e del Nuovo Testamento nei registri laterali e pure dal ciclo dei mesi nel porticato adiacente l'ingresso (Figg. 4, 5) ${ }^{27}$. Alcuni cicli, nel mettere in relazione i mesi con le Storie di Cristo o con altre raffigurazioni dal contenuto escatologico, si trasformavano in veri e propri calendari allegorici dell'era dell'Incarnazione, una sorta di almanacchi liturgici che scandivano il tempo dell'uomo in attesa della seconda venuta di Cristo, seguendo, tra l'altro, un programma assai caro al progetto di riforma religiosa portato a termine durante il XII secolo ${ }^{28}$.

Secondo Francovich l'uso di raffigurare i mesi su capitelli si fa risalire a consuetudini bizantine, dal momento che quella tradizione si trovava esclusivamente in zone

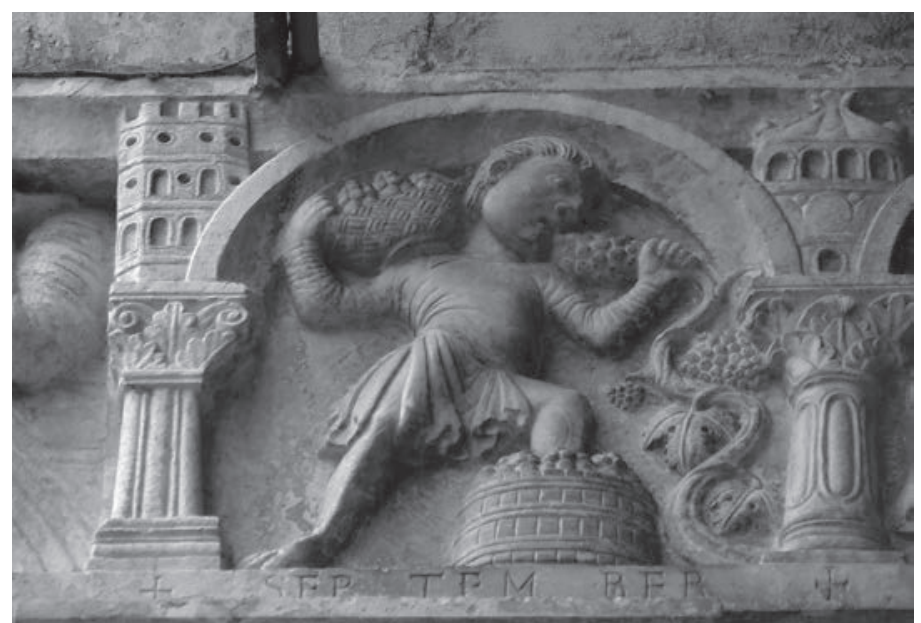

Fig. 5. Verona, San Zeno, portale maggiore, particolare del mese di Settembre (Foto Autore)

permeate di influenze orientali ${ }^{29}$. Il significato del valore 'parlante' della raffigurazione dei mesi variava anche dalla tipologia di abbinamento figurativo. Ad esempio, se esposto in coppia con lo zodiaco, la fusione iconografica dal forte portato allusivo poteva riferirsi convenzionalmente all'avanzare del tempo e poteva anche reinterpretare in una versione semplificata lo stretto legame tra terra e cielo e tra il microcosmo umano e l'universo ${ }^{30}$. Tale impaginato narrante si prestava al racconto della vita sulla terra dell'uomo e pure alle inevitabili correlazioni col sacro, in entrambi i casi con lo scopo di rappresentare lo scorrere del tempo in funzione del sacrificio di Cristo $^{31}$. La ciclicità di mesi corrispondeva alla periodicità liturgica, che si riproponeva ogni anno e che scandiva la ferialità quotidiana, le domeniche e le feste religiose, come l'Annunciazione, la Pasqua, la Pentecoste, l'Assunzione e la Natività.

I cicli raffigurativi di quella scansione temporale con un chiaro valore rituale elaborati tra la fine del secolo XI e l'inizio del successivo erano resi generalmente attraverso una modulazione narrante sintetica e improntata su una specifica valenza figurativa del messaggio; quelli successivi, sino al pieno Duecento, assunsero peculiarità più realistiche coerentemente anche a una diffusione della interpretazione del vero marcatamente federiciana, inscenando dunque un tempo piuttosto materiale ${ }^{32}$. Si noti, ad esempio, come nel complesso schema iconografico del portale della cattedrale di Trani della fine del XII secolo si faccia una breve menzione a uno solo dei mesi, quello di gennaio, applicato in uno scomparto dell'anta interna destra dell'ingresso ${ }^{33}$. La peculiarità della sua presenza rimane legata all'estremo sunto

\footnotetext{
${ }^{26}$ GIURISATO, Il cenobio di Benedetto, p. 51.

27 D. DE FRANCOVICH, Contributi alla scultura romanica veronese, in Rivista dell'Istituto Nazionale di Archeologia e Storia dell'Arte, IX (1941/1942), p. 102-147; A. CALZONA, Niccolò e Verona. La facciata e il protiro di San Zeno in Nicholaus e l'arte del suo tempo, II, Ferrara, 1985, p. 441-489; I. DEDONI, La memoria dell'antico nei cantieri romanici della Basilica di San Zeno, in Annuario storico zenoniano, 16 (1999), p. 25-52; V. PACE, Le sculture di facciata di San Giovanni in Venere: una diramazione veronese in Abruzzo e il loro problematico contesto, in A. C. Quintavalle (a cura di), Medioevo: arte lombarda, Atti del Convegno Internazionale di studi (Parma, 26-20 settembre 2001), Milano, 2004, pp. 476-487; G. VALENZANO, L'iconographie du portail de SaintZenon à Verone et sa façade, in Les Cahiers de Saint-Michel de Cuxa, 45 (2014), p. 217-230; F. STROPPA, Natura e figura nella rappresentazione dei mesi, in A.C. Quintavalle (a cura di), Medioevo. Natura e figura, p. 444-462.

${ }_{28}^{28}$ A. TRIVELLONE, L'arte e la riforma ecclestiastica tra XI e XII secolo, in U. Eco (a cura di), Il Medioevo. Cattedrali, cavalieri, città, II, Milano, 2011, p. 605612; S. RICCIONI, Il mosaico absidale di S. Clemente a Roma "exemplum" della chiesa riformata, Spoleto, 2006.

${ }_{29}$ G. DE FRANCOVICH, Benedetto Antelami: architetto e scultore e l'arte del suo tempo, Milano, 1952, p. 441, nota 252.

${ }^{30}$ STROPPA, Natura e figura nella rappresentazione dei mesi, p. 447.

${ }^{31}$ Ibidem.

${ }^{32}$ Ibidem.

33 M. S. CALÒ MARIANI, Scultura pugliese del XII secolo. Proto magistri tranesi nei cantieri di Barletta, Trani, Bari e Ragusa, in Studi di Storia dell'Arte in memoria di Mario Rotili, I, Napoli, 1984, p. 177-191; P. BELLI D'ELIA, L'officina barese: scultori a Bari nella seconda metà del XII secolo, in Bollettino d'Arte, VI, 69 (1985), 27, p. 13-48.
} 


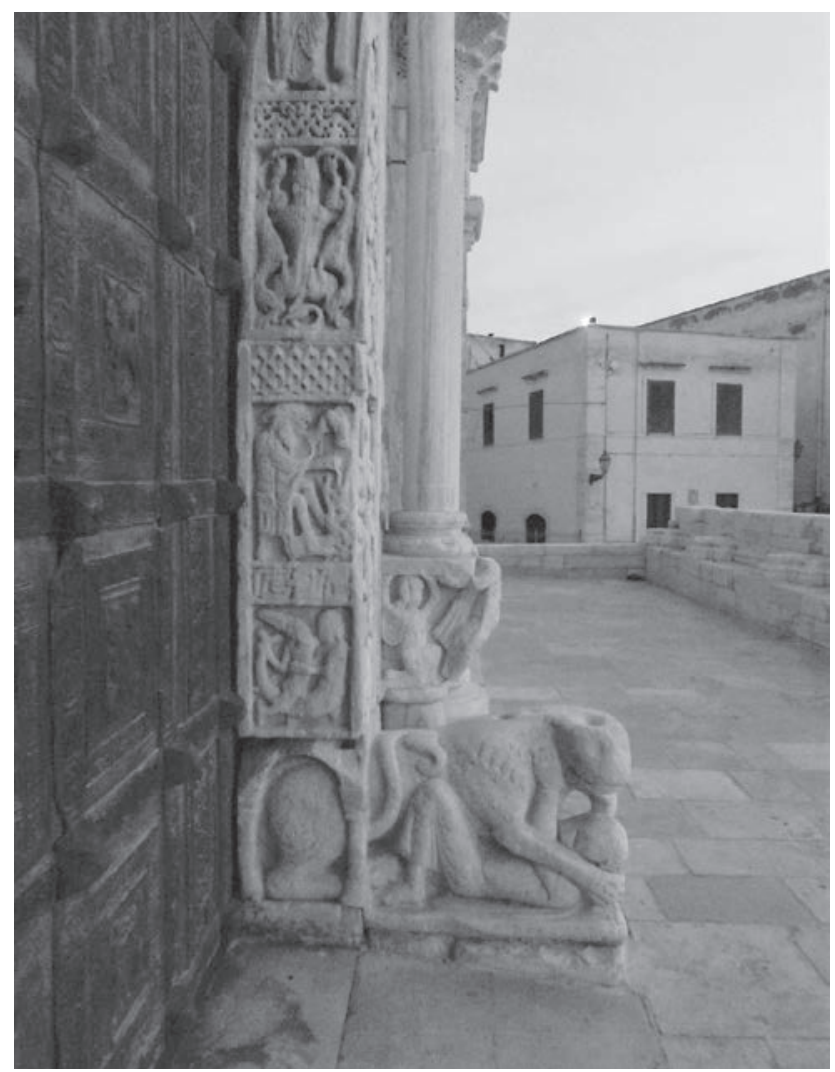

Fig. 6. Trani, cattedrale, portale maggiore, particolare del mese di Gennaio (Foto Autore)

illustrativo e anche alla sua unicità che sottende una precisa scelta comunicativa, dipendente da un gioco di sintesi perfettamente riconoscibile (Fig. 6). D'altro canto, nella più ricercata manifestazione pittorica del XIII secolo avanzato, la possibilità di materializzare effettivamente un'immagine diviene un espediente affabile di un certo gusto raffinato, che bene si evidenzia, ad esempio, nella messa in scena di un calendario nell'oratorio di San Pellegrino a Bominaco del cosiddetto Maestro miniaturista, ad uso liturgico della comunità monastica con la raffigurazione cortese dei mesi alternati ai segni zodiacali (Fig. 7) ${ }^{34}$.

Se nel Meridione la presenza di quel ciclo costituisce spesso un aspetto figurativo di un programma ben più ampio legato a contesti religiosi, nel corso del XIII secolo nel Nord Italia quella presenza fiorisce anche in contesti prettamente civili o comunque inseriti nel tessuto cittadino, come si registra nel ciclo del portale maggiore della basilica di San Marco a Venezia; proprio nella realtà lagunare i mestieri svolsero l'importante funzione di disciplinamento della vita sociale nel contesto urbano, dedicata al commercio e alle attività mercantili ${ }^{35}$. Legato al cantiere veneziano è indubbiamente il portale della cattedrale di Trogir per questioni iconografiche

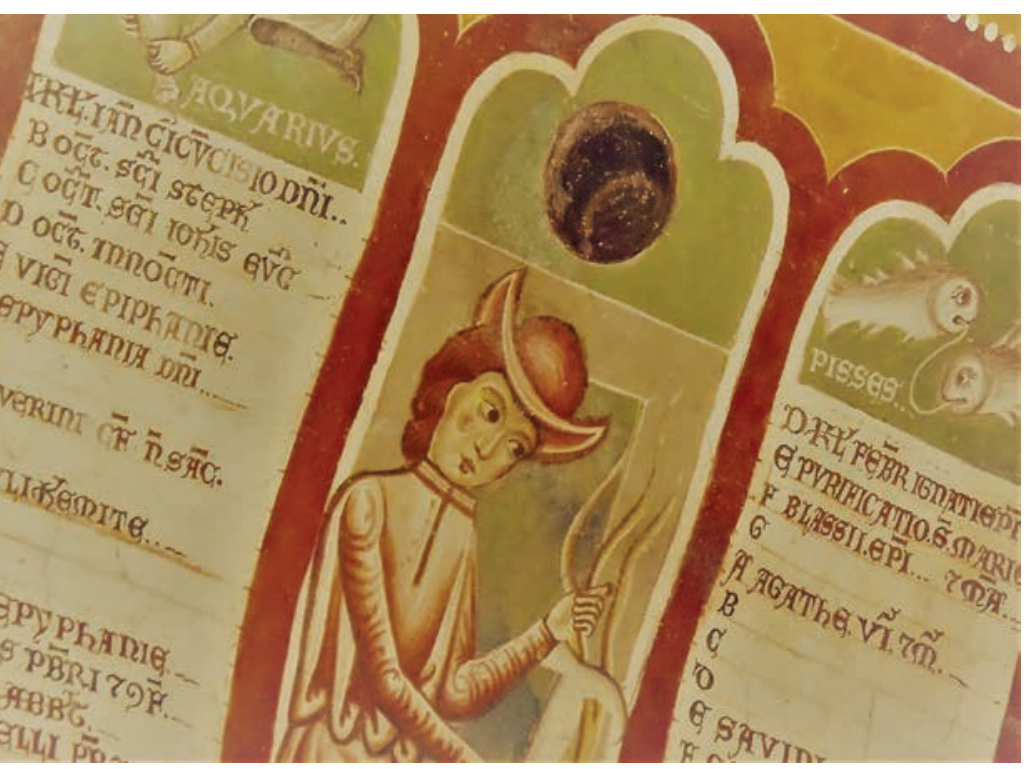

Fig. 7. Bominaco, oratorio di San Pellegrino, affresco, particolare del calendario (Foto Autore)

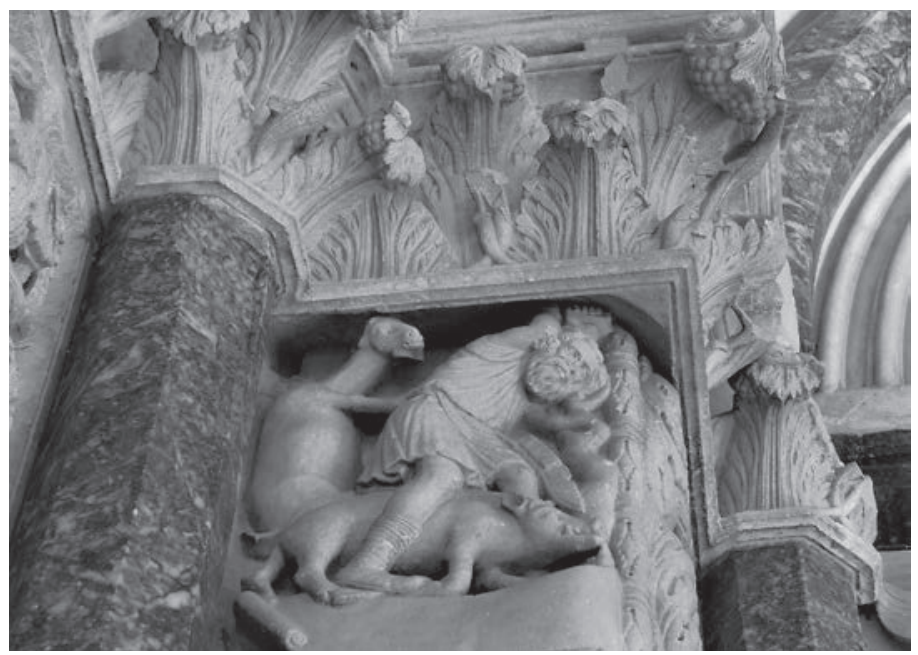

Fig. 8. Trogir, cattedrale, portale maggiore, particolare del ciclo dei Mesi (Foto Autore)

e pure per rimandi stilistici ${ }^{6}$. In questa complessa macchina decorativa trova posto anche la raffigurazione del ciclo dei mesi negli stipiti del portale, scolpiti da una generazione successiva al magister Radovan che concluse l'ingresso nel 1240. Alla stregua dei campioni di riferimento pittorici menzionati, è chiaro che anche la descrizione stilistica adottata nei rilievi dalmati aderisce a un processo di riepilogo iconografico denso di particolarismi realistici, come si può notare nella raffigurazione del mese di gennaio, in cui un uomo barbato e dai folti capelli è colto nell'atto di uccidere un maiale che tiene stretto tra le gambe (Fig. 8).

${ }_{34}$ G. MATTHIAE, Pittura medioevale abruzzese, Milano, 1969, p. 50-51; V. PACE, Precisazioni sugli affreschi dell'Oratorio di San Pellegrino a Bominaco, in Commentari, 21 (1970), p. 291-297; V. BRANCONE, Complementi iconografici per il calendario dipinto dell'oratorio di San Pellegrino a Bominaco, in Arte medievale, 3, 2004 (2005), 2, p. 75-108; M. DELLA VALLE, Osservazioni sui cicli pittorici di San Pellegrino a Bominaco e di Santa Maria ad Cryptas di Fossa in Abruzzo, in Acme, 59, 2006 (2007), 3, p. 101-158; V. LUCHERINI, Una proposta 'romana' per gli affreschi duecenteschi di San Pellegrino a Bominaco (L'Aquila), in Napoli Nobilissima, 5, 1 (2000), 5/6, p. 163-188; V. LUCHERINI, L'abbazia di Bominaco in Abruzzo. Organizzazione architettonica e progetto decorativo (XI-XIII secolo), Roma 2016.

35 G. TIGLER, Il portale maggiore di San Marco a Venezia: aspetti iconografici e stilistici dei rilievi duecenteschi, Venezia, 1995 (Memorie classe di Scienze morali, lettere e arti, 59). Per tutti gli altri celebri casi del duomo di Ferrara, del battistero di Parma, della cattedrale di Arezzo si rimanda a B. Giovannucci Bigi, G. Sassu (a cura di), Il Maestro dei Mesi e il portale meridionale della cattedrale di Ferrara: ipotesi e confronti, Giornata di studi (Ferrara, 1 ottobre 2004), Ferrara, 2007, con bibliografia precedente.

${ }^{36}$ G. TIGLER, La porta dei Mesi del Duomo di Ferrara e le sue derivazioni in Arezzo, Fidenza e Traù, in Il Maestro dei Mesi e il portale meridionale della cattedrale di Ferrara, p. 71-101; B. RADOSLAV, Il Maestro Radovan e l'incompiuto portale romanico della cattedrale di Trogir, in Ravennatensia, 24 (2009), p. 123-313; A. CALZONA, Alcune note sulla cronologia del battistero di Parma e la possibile presenza di Radovan tra i maestri della bottega antelemica, in M. 


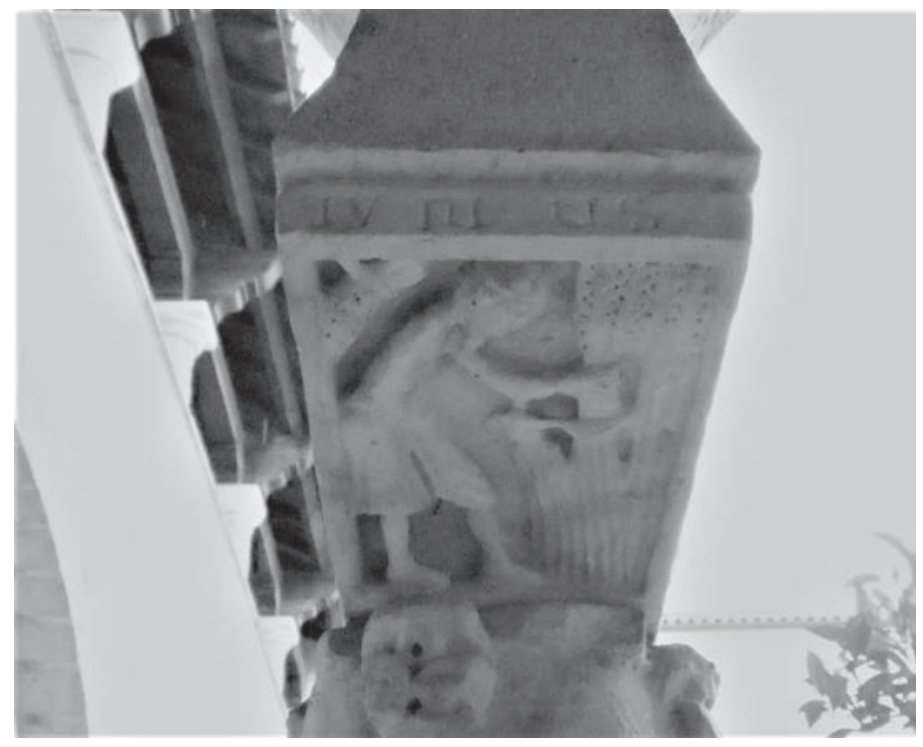

Fig. 9. Benevento, Santa Sofia, chiostro, capitello con il mese di Giugno (Foto Autore)

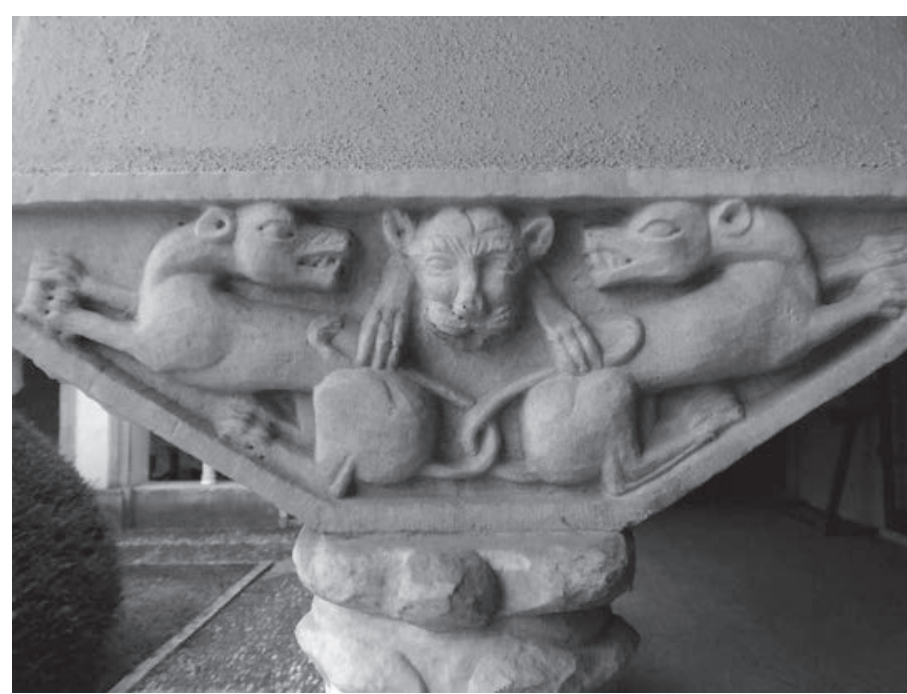

Fig. 11. Benevento, Santa Sofia, chiostro, capitello con animali (Foto Autore)

I mesi ancora conservati nel chiostro sofiano a Benevento sono sette e la loro rappresentazione ha inizio nei due capitelli dell'unica trifora del chiostro che forma con la successiva quadrifora un gomito che, assecondando la sporgenza della chiesa alla quale esso è addossato, ne interrompe la pianta quadrangolare. Sui lati brevi dei capitelli si trovano i mesi di giugno (Fig. 9) e luglio nelle tradizionali occupazioni della mietitura e della trebbiatura, agosto con la raccolta della frutta, settembre che pigia i grappoli d'uva in una scenografia di vendemmia, poi ottobre con due uomini intenti al lavoro campestre e novembre con la semina (Fig. 10) e infine dicembre con il maiale ucciso sulle spalle ${ }^{37}$. A dispetto della restituzione realistica esaminata negli esemplari precedenti, qui si assiste a processo di semplificazione stilistica che implica pure una facilitazione della comprensione del

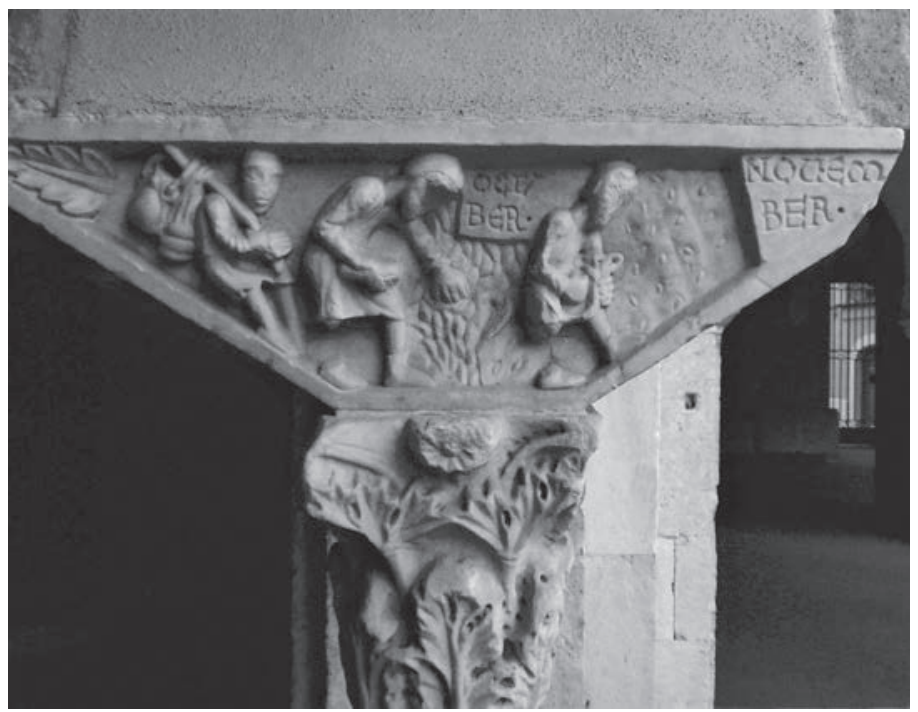

Fig. 10. Benevento, Santa Sofia, chiostro, capitello con i mesi di Ottobre e di Novembre (Foto Autore)

messaggio figurativo, favorita dalla descrizione epigrafica del mese corrispondente.

Tracciando una mappatura virtuale di altri esemplari di ciclo dei mesi conservati nel Sud Italia, citando quelli più noti esposti nei portali della cattedrale di Trani e di Sessa Aurunca ${ }^{38}$, si nota una netta divergenza nella scelta iconografica e stilistica, tale da permettere di avanzare forse più timide assonanze con il panorama lombardo della fase post antelemica ${ }^{39}$. Al maestro dei Mesi di Benevento andrebbero ricondotti pure i tre capitelli della terza quadrifora del lato nord, con animali che si azzannano (Fig. 11), sirene, figure di cacciatori inframmezzate da acanti di forte accezione abruzzese. Bertaux, dopo aver rintracciato affinità con la plastica campana del XII secolo, specialmente con il repertorio scultoreo dell'abbazia di Montevergine e con quello della cripta del duomo di S. Agata dei Goti, li considerò parte di un gruppo di opere eseguito da artisti lombardi che pure avevano lavorato in contesti meridionali, quali, ad esempio, i portali della cattedrale di Barletta ${ }^{40}$.

Secondo la proposta più convincente di Cochetti si trattò invece di artisti locali ritardatari che rielaborarono motivi iconografici e soprattutto stilistici molto tempo dopo ${ }^{41}$; dovette trattarsi di una bottega di lapicidi autoctoni che però risentì anche le influenze del vicino laboratorio della Capitanata e dell'Abruzzo, se si pongono a confronto alcuni dettagli sofiani con le sculture allineate lungo l'esterno della cattedrale di Troia e con quelle che decorano le finestre absidali di Santa Maria del Lago a Moscofo, entrambe ancorate alla fine del XII secolo ${ }^{42}$ (Figg. 12, 13). Ulteriori comparazioni si possono stendere anche con la produzione scultorea legata ai cantieri attivi proprio in quel momento a Bari, riferendomi in particolar modo a un rilievo con due scimmie con le code intrecciate sotto i corpi secondo una tipica consuetudine araldica, proveniente dalla chiesa di S. Pelagia ${ }^{43}$.

Cepetić, D. Dujmović (a cura di), Art history, the future in now. Studies in honor of professor Vladimir P. Goss, Rijeka, 2012, p. 158-174; M.C. ROSSI, L'abbazia di San Giovanni in Venere. Un laboratorio per la scultura in Abruzzo tra XI e XIII secolo, Pescara, in stampa.

37 COCHETTI, La decorazione plastica del chiostro di Santa Sofia, p. 19.

${ }^{38}$ Ibidem, e si ricorda anche quello a mosaico della cattedrale di Otranto.

${ }^{39}$ Ivi, p. 20

${ }^{40}$ È BERTAUX, L'art dans l'Italie méridionale. De la fin de l'Empire romain à la conquête de Charles d'Anjou, II, Paris, 1903 (Roma 1968), p. 472-476.

${ }^{41}$ COCHETTI, La decorazione plastica del chiostro di Santa Sofia, p. 26.

${ }^{42}$ BELLI D’ELIA, La Puglia, p. 406-430; F. GANDOLFO, Scultura medievale in Abruzzo in età normanno-sveva, Pescara, 2004, p. 76-83.

43 BELLI D'ELIA, La Puglia, p. 123. 


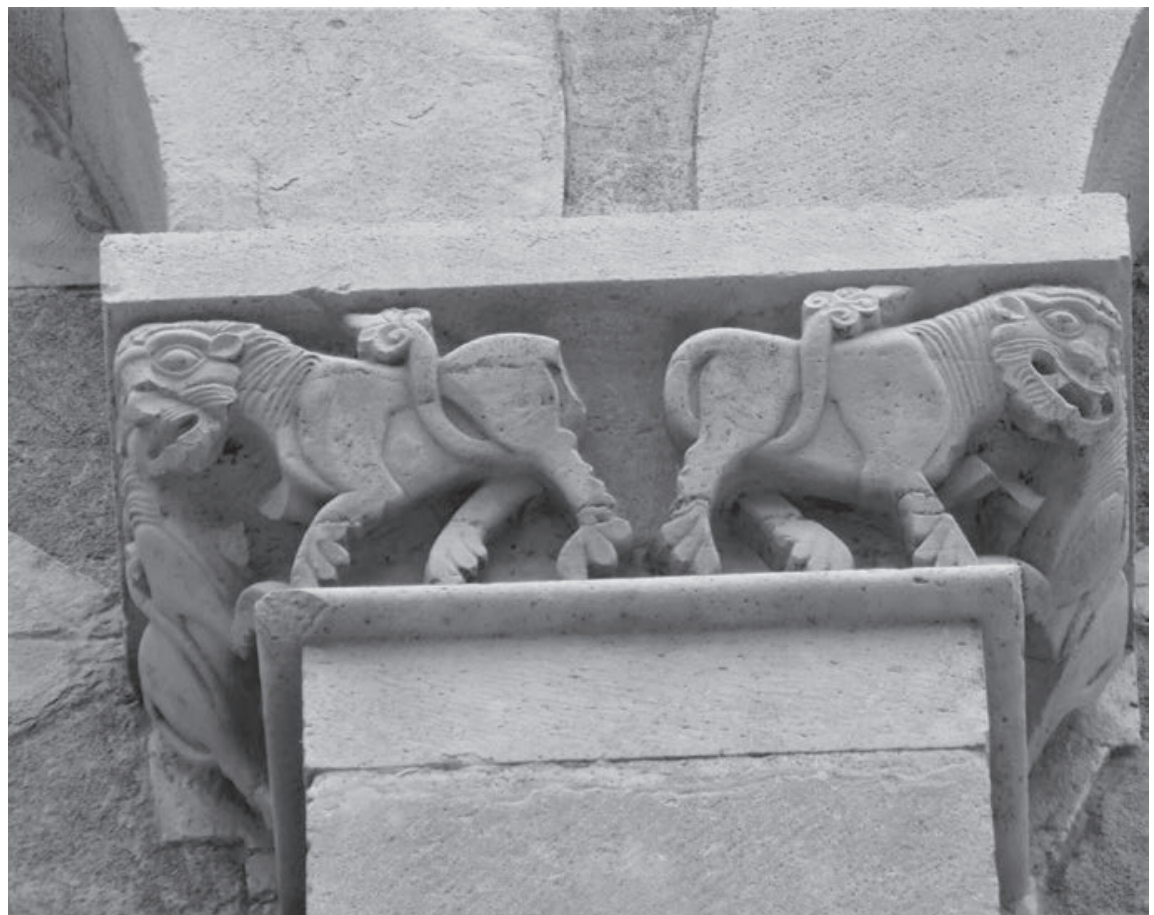

Fig. 12. Troia, cattedrale, esterno, particolare (Foto Autore)

Per quel che riguarda, invece, uno dei più celebri capitelli scolpiti sofiani, ritraente due cavalieri in lotta non vi è alcun dubbio sulla paternità sia iconografica sia stilistica del pendant conservato nel chiostro della vicina abbazia di Montevergine ${ }^{44}$. E proprio a proposito dello stile, non è possibile non tenere a mente i rapporti artistici, dispiegati nei termini di circolazione di modelli e di maestranze, tra la Sicilia e la Campania che nel corso degli anni ottanta del XII secolo si fecero più intensi; in tale processo di condivisione pluriculturale rientra anche la decorazione sofiana che, tuttavia, rimase per lo più indipendente da quel linguaggio tendente alla resa spaziale del rilievo e all'attenzione alla qualità mimetica ${ }^{45}$. A spezzare la lancia a favore di un arricchimento compositivo dell'insieme è il nodo che si trova a metà di una colonnina ${ }^{46}$ (Fig. 14). Quello della colonna annodata, o

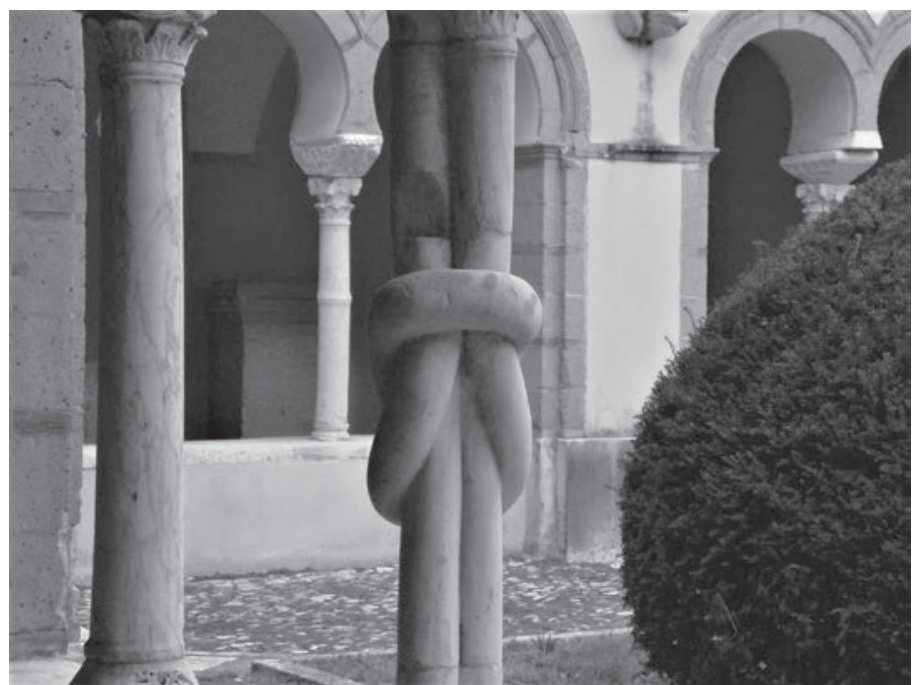

Fig. 14. Benevento, Santa Sofia, chiostro, colonna ofitica (Foto Autore)

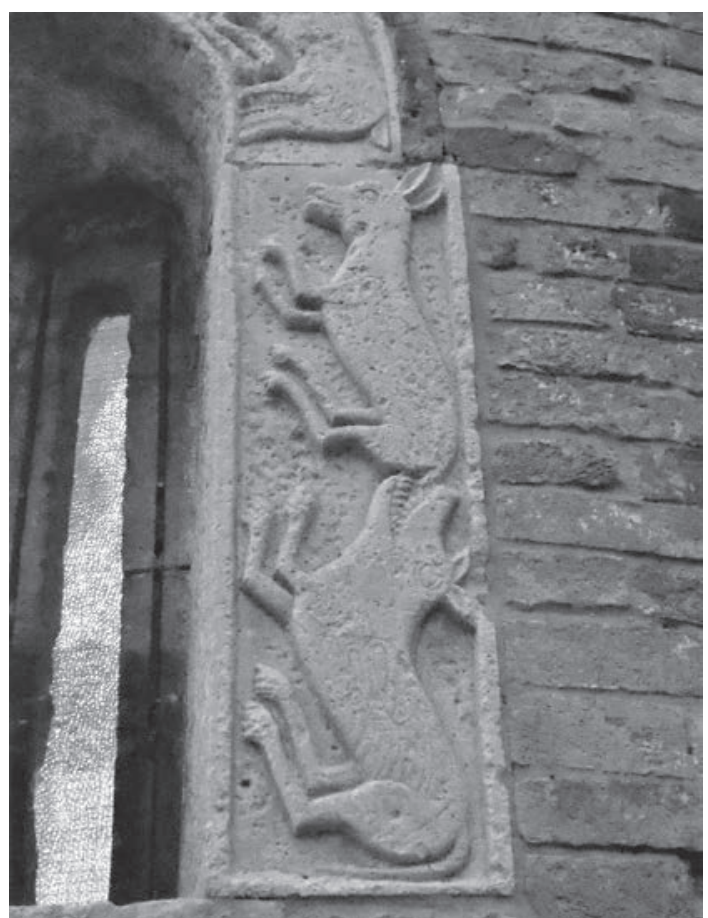

Fig. 13. Moscufo, Santa Maria del Lago, abside, particolare (Foto Autore)

ofitica, è un motivo abbastanza diffuso nell'architettura monastica specialmente dell'Italia settentrionale e che trova il riferimento più famoso in quello dell'abbazia di Chiaravalle. Si tratta evidentemente dell'ennesimo riferimento simbolico e precisamente alla doppia natura umana e divina di Cristo. Vorrei mostrare in questa sede un ricordo al proposito, il bellissimo esemplare in marmo bicromo, datato al XII secolo, conservato nel Museo di Arte Sacra di Zadar, appartenente al mobilio liturgico, probabilmente un'iconostasi, della chiesa medievale di Santa Maria di Zadar (Fig. 15).

Sinora la letteratura che si è occupata della decorazione plastica del chiostro beneventano ha assegnato ai capitelli una datazione alla prima metà del XII secolo ${ }^{47}$, malgrado la presenza di un'iscrizione sul primo capitello del lato nord

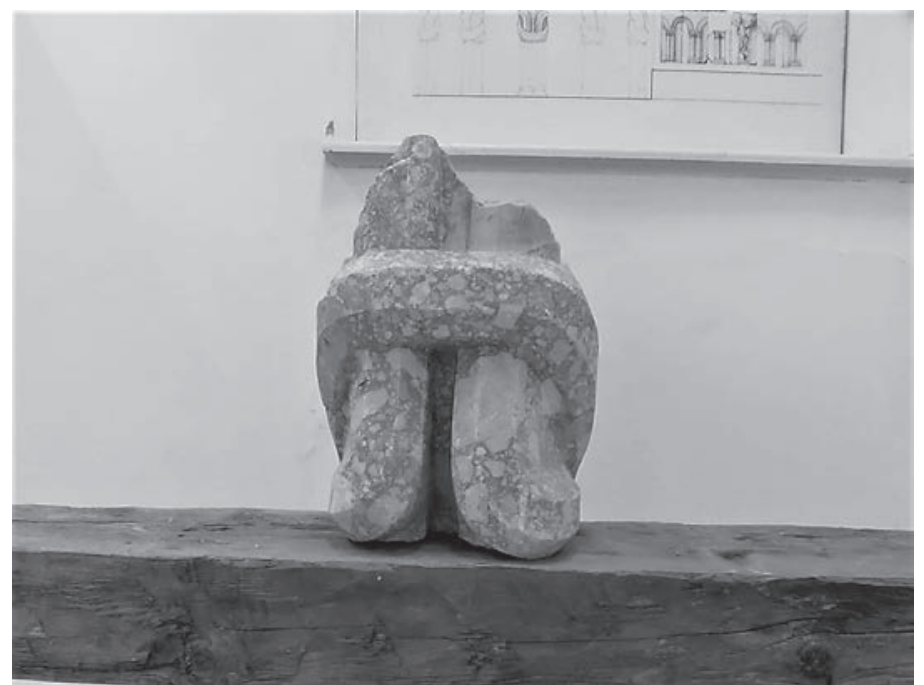

Fig. 15. Zadar, Museo di Arte Sacra, colonna ofitica (Foto Autore)

${ }_{44}$ G. MUOLLO, Capitelli a stampella, in P. Leone de Castris (a cura di), Il Museo abbaziale di Montevergine, Catalogo delle opere, Napoli, 2016, p. 41-43.

${ }_{45}$ COCHETTI PRATESI, In margine ad alcuni recenti studi sulla scultura medievale nell'Italia meridionale. Sui rapporti tra la scultura campana e quella siciliana, in Commentari. Rivista di critica e storia dell'arte, IV (1970), p. 255-290.

${ }^{46}$ GALASSO, Il chiostro di Santa Sofia a Benevento, p. 20.

${ }_{47}$ BERTAUX, L'art dans l'Italie méridionale, p. 476; P. TOESCA, Storia dell'arte italiana. Il Medioevo, II, Torino, 1927, p. 849; L. COCHETTI, La decorazione plastica del chiostro di Santa Sofia, p. 17. 


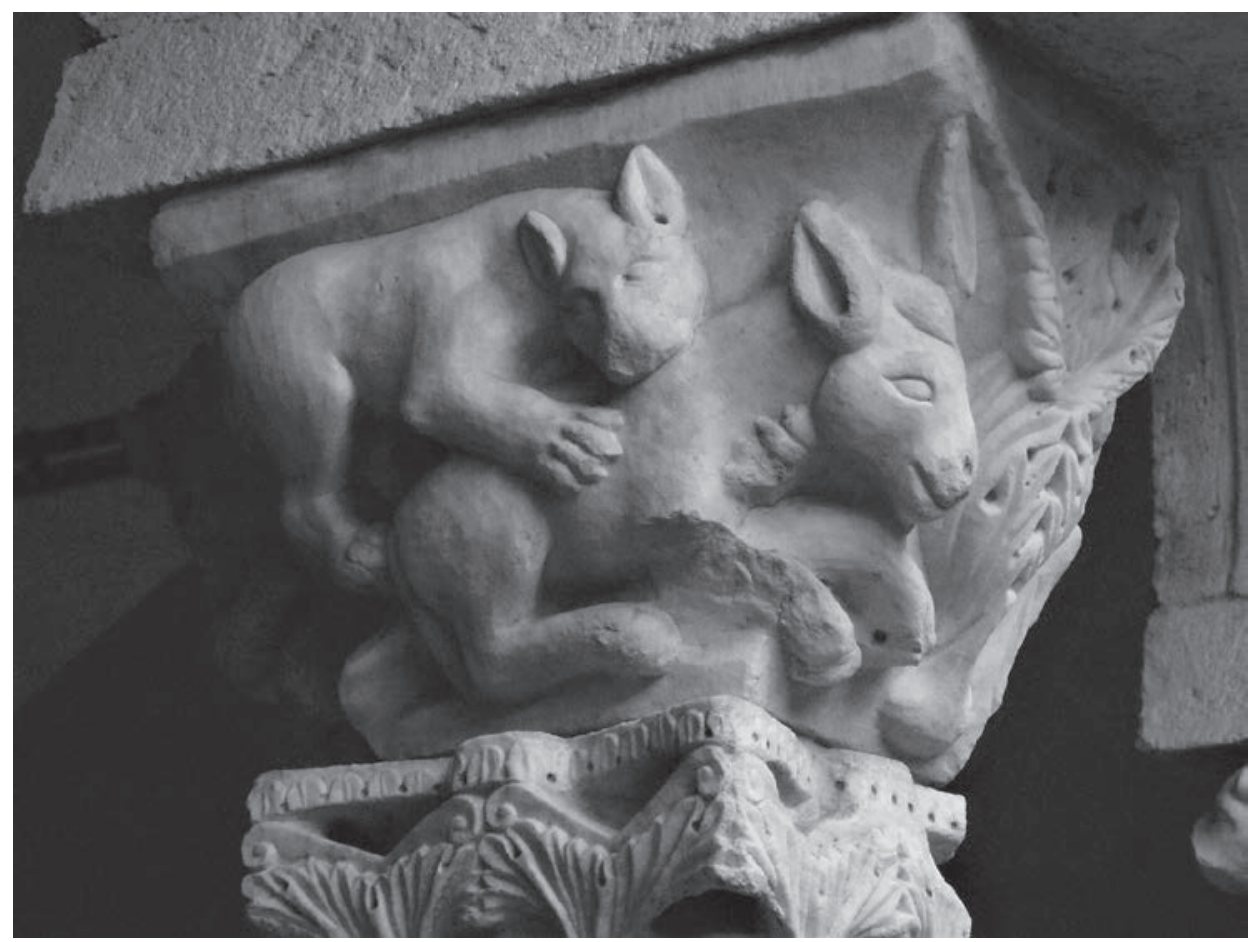

Fig. 16. Benevento, Santa Sofia, chiostro, capitello con lotta tra belve (Foto Autore)

cevano $^{53}$. Nella storia artistica medievale del Meridione italiano si ricorda come snodo fondamentale il 1071, l'anno della consacrazione della basilica abbaziale di Montecassino, nella quale erano confluite maestranze di estrazione culturale e provenienza diversa, amalfitana, lombarda e bizantina ${ }^{54}$. Di lì a poco questa sintesi entrò a fa parte del linguaggio dell'arte meridionale tout court, valorizzata e celebrata attraverso l'impiego di spolia, presenti sia a Montecassino sia nel duomo di Salerno, consacrato nel 1085 e che condivideva con il cenobio benedettino la politica ideologica di ritorno al modello 'classico', che pure si ritrova nella decorazione del chiostro sofiano ${ }^{55}$.

Per Francesco Gandolfo lo stile dei capitelli di Benevento trova una forte corrispondenza in alcuni a stampella riutilizzati nella cripta della cattedrale di Sant'Agata dei Goti e in due conservati nell'abbazia di Montevergine, consacrata nel 1182, specialmente, come si diceva, nel capitello coi cavalieri che duellano ${ }^{56}$.

che cita il nome di Johannis, a cui però non si è riuscito a far corrispondere un sicuro personaggio 'datante', salvo un Giovanni firmatario di un documento del 1151, forse l'abate Giovanni IV, in carica dal 1142 al $1176^{48}$ o Giovanni il Grammatico, abate nel $1119^{49}$. Di parere contrario era Cochetti che proprio per il carattere di disomogenità dell'insieme scultoreo e pure dell'impiego vario di materiale di spoglio ${ }^{50}$, ritenne che l'impresa fosse stata portata a termine in almeno due fasi, se non tre e che quella più tarda avesse interessato quasi esclusivamente il lato nord ${ }^{51}$. Si tratta peraltro di una consuetudine diffusa a Benevento, quella del ricorso a capitelli più antichi come reimpiego, molti dei quali conservati oggi nel Museo del Sannio ${ }^{52}$.

Per quel che concerne la datazione non possono apportare valutazioni aggiuntive neppure i capitelli inferiori non figurati, imitazioni o rielaborazioni di modelli classici. Le colonnine, le basi, i capitelli, i pulvini sono infatti tutti diversi, tanto da rappresentare una gamma di stili; tanto più che il periodo longobardo non ci diede un'arte propria, ma si appropriò quella che gli artisti delle contrade produ-
Tuttavia, alla luce delle evidenze morfologiche chiaramente condizionate dalla plastica di un territorio ampio ed esteso da Benevento alla Capitanata con notevoli ascendenze abruzzesi e molisane, si ritiene di rintracciare in loco l'origine della bottega, trovando un utile confronto tra alcuni capitelli sofiani e le sculture che decorano l'architrave del portale della chiesa beneventana di San Bartolomeo, in un momento collocabile nella seconda metà del XII secolo57. Non bisogna dimenticare che il XII secolo fu il periodo più florido dell'abbazia ${ }^{8}$, quando si assistette anche al maggiore successo di un repertorio plastico particolarmente sperimentato nei cantieri abruzzesi e pugliesi dalla seconda metà dello stesso secolo. Eppure la bottega attiva nel chiostro sofiano accolse retaggi stilistici ancora fortemente implicati nella storia della scultura del Mezzogiorno centro-meridionale tra XI eXII secolo ${ }^{59}$; purtuttavia l'adesione a un codice figurativo superato e, se si vuole, arcaico, non indica necessariamente un'inclinazione al ritardo nel recepire impulsi innovativi, quanto piuttosto una consapevole scelta di attenta elaborazione e di altrettanta valutazione dello scarto.

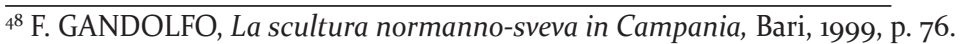

${ }^{49}$ A. MEOMARTINI, Benevento con 144 illustrazioni, Bergamo, 1909, p. 112; A. ZAZO, L'abate Giovanni IV alter conditor del chiostro di Santa Sofia a Benevento, in Samnium, 10 (1937), p. 238-239; M. ROTILI, L'arte nel Sannio, Benevento, 1952, p. 78.

50 M. COSTAGLIOLA, Nuove acquisizioni sui restauri novecenteschi della chiesa longobarda di Santa Sofia a Benevento, in Apollo. Bollettino dei Musei Provinciali del Salernitano, XVIII (2002), p. 32-74: 32.

${ }^{51}$ COCHETTI, La decorazione plastica del chiostro di Santa Sofia, p. 18.

${ }^{52}$ M. ROTILI, L'eredità dell'antico a Benevento dal VI all'VIII secolo, in Napoli Nobilissima, XIV, 4 (1975), p. 121-128: 125.

53 MEOMARTINI, Benevento con 144 illustrazioni, p. 111.

54 PACE, Arte medievale in Italia, p. 17-18.

55 G. CHIERICI, Il duomo di Salerno e la chiesa di Montecassino, in Rassegna storica salernitana, I (1937), pp. 95-109; A. SCHIAVO, Montecassino. Affinità stilistiche tra la chiesa cassinese di Desiderio e quella salernitana di Alfano I, Atti del II Convegno Nazionale di Storia dell'Architettura (Assisi 1937), Roma, 1939, p. 159-176.

${ }^{56}$ GANDOLFO, La scultura normanno-sveva, p. 41-43.

57 Ivi, p. 46.

$5^{8}$ E. GALASSO, Inediti capitoli di riforma per S. Sofia di Benevento, in Samnium, 3-4 (1969), p. 11-113, 116.

59 Cfr. P. BELLI D’ELIA, Alle sorgenti del romanico. Puglia XI secolo, Bari, 1975, specialmente i cantieri attivi tra la fine del secolo XI e l’inizio del Mille in Capitanata e in Terra di Bari.
} 
Il repertorio decorativo è costantemente rivolto alla commemorazione dell'antico, come si può notare in un capitello su cui è raffigurata la lotta tra due belve (Fig. 16), tanto da divenire il filo conduttore del programma scultoreo generale, che si eleva a manifesto ideologico dell'esposizione. È per questa ragione che l'insieme di diversi contenuti, vetero e neotestamentari, appartenenti al genere cavalleresco, alla rubrica dedicata al ciclo dei mesi, alla citazione dell'antico e così via, confluisce coerentemente nell'intenzione didascalica della comunicazione rivolta alla comunità monastica. 